\title{
Dynamic Spectrum Access and Coexistence Experiences Involving Two Independently Developed Cognitive Radio Testbeds
}

\author{
Nolan, K.E., Sutton, P.D., Doyle, L.E. \\ Centre for Telecommunications Value-Chain Research (CTVR) \\ University of Dublin, Trinity College \\ Dublin, Rep. of Ireland \\ Telephone: (+353) 1-8968443 \\ Fax: (+353) 1-8968442 \\ keithnolan@mee.tcd.ie, suttonpd@tcd.ie, ledoyle@tcd.ie
}

\author{
Rondeau, T.W., Le, B., Bostian, C.W. \\ Centre for Wireless Telecommunications (CWT) \\ Virginia Tech \\ Blacksburg, VA, USA \\ Telephone: (+1) 540-231-5096 \\ Fax: (+1) 540-231-3004 \\ trondeau@vt.edu, binle@vt.edu, bostian@vt.edu
}

\begin{abstract}
The Centre for Telecommunications Value-Chain Research (CTVR) and the Center for Wireless Telecommunications (CWT) are carrying out joint research work investigating the potential of different software-defined radio (SDR) and cognitive radio (CR) systems that can coexist in common frequency bands. This paper describes the independently developed dynamic spectrum access test beds used in a practical coexistence experiment. An initial analysis of actual coexistence experiences involving a primary user and a secondary opportunistic spectrum user in a common frequency band is also presented. The results in this paper include an analysis of a worst case scenario where the primary user and secondary opportunistic user are coexisting with no guard bands separating each other. The experimental results showed that the primary user experienced zero packet loss when guard bands separated the primary and secondary services. Additionally, when no guard bands were used and the spectrum segment was maximally used over the geographical area involved in the experiments, the signal to noise and interference ratio (SNIR) needed to be adjusted to $20 \mathrm{~dB}$ by modifying the secondary user transmissions in order to minimise the interference experienced by the primary user.
\end{abstract}

\section{INTRODUCTION}

The use of cognitive functionality in wireless telecommunication systems and networks presents new and exciting possibilities for improving the way information is conveyed between nodes. In addition, this technology also paves the way for new wireless applications that can exploit the abilities of different cognitive radio architectures to inter-operate, coexist, and work together as a team within this network. In order to demonstrate and evaluate these possibilities, the Centre for Telecommunications Value-Chain Research (CTVR) and the Center for Wireless Telecommunications (CWT) are undertaking a joint collaborative research effort investigating these possibilities. This paper builds on previous work carried out as part of this collaborative effort [1], [2] and details further outcomes of coexistence experiments in the $2.4 \mathrm{GHz}$ industrial, scientific and medical (ISM) frequency band.

The term whitespace denotes spectrum that is unused at any particular point of observation. The main objective of the coexistence experiments was to exploit unused spectrum adjacent to a narrowband primary user frequencies and to explore the effect of reducing the guard bands between the primary user and opportunistic secondary users. These systems have the capability to accomplish the experimental objectives and to investigate the effects of dynamic spectrum access on legacy systems because each of the systems have been built with reconfigurability and cognitive functionality in mind. In addition, these abilities can also be deactivated allowing either system to assume the role of a fixed, non-adaptive legacy system. It may be useful therefore to first explain what the authors mean by the terms cognitive functionality, cognitive radio, and cognitive networks.

\section{A. Cognitive Radio and Networks}

Cognitive radio can be described as a node in a network with the abilities to form an awareness of its environment and context, make decisions and inferences from this information combined with knowledge of the user's objectives, act in a manner that attempts to accomplish the user's objectives, and finally learn from these experiences for possible use in the future [3], [5]. This cognitive functionality may have an influence on all or many of the layers in a communications stack and is not just limited to the Physical Layer (PHY) only. Both of the systems used for the coexistence experiments are capable of being reconfigured and using information derived from spectrum measurements in order to exploit available spectrum and to avoid frequencies in use by primary users.

\section{B. Architecture Overviews}

This section outlines the coexistence scenario that was explored, leading to the experiences reported in this paper. This scenario involved two cognitive radio systems with different architecture designs and cognitive capabilities. The Center for Wireless Telecommunications assumed the role of a primary user and the Centre for Telecommunications ValueChain Research (CTVR) assumed the role of an opportunistic 
secondary user. This scenario essentially explored the ability of these two different CR architectures, using different wireless communication schemes, to coexist on non-interfering and interfering bases. The purpose of this being to refine the mechanism used to exploit spectrum opportunities (unused or under-utilised whitespace frequency spectrum).

The first architecture involved in these tests is a GNU Radiobased platform where the parameters of the communications stack (the knobs) can be adjusted using a genetic algorithmbased cognitive control mechanism using information derived from performance metrics (the meters). This CR architecture is designed and implemented by CWT, Virginia Tech, USA [4], [8]. The second architecture design is a maximallyreconfigurable software radio system is called the Plastic Project [6], designed and implemented by CTVR, University of Dublin, Trinity College, Rep. of Ireland. The parameters and structure of the entire communication stack can be dynamically reconfigured according to a less complex cognitive control implementation.

\section{COEXISTEnCE}

Coexistence, as referred to in this paper, is the ability of two or more nodes/entities to share a common frequency band. Cognitive radio coexistence can help to increase the efficiency of how spectrum is used, where two or more services can share a common frequency band. The term interference-free coexistence is the term denoting an ability for two or more entities to share a common space without causing interference to each other. In this case, the two or more entities are wireless transmissions occupying a common frequency spectrum segment. Exclusive spectrum usage rights for some currently licensed frequency bands can support more services if these secondary (opportunistic) users can operate on an interferencefree basis with the primary/legacy user. This is another key area in cognitive radio and dynamic spectrum access research.

\section{CTVR AND CWT COGNITIVE RADIO ARCHITECTURES}

This section provides a brief overview of the key features of the two different architectures used for the experiments in this paper. The coexistence experiments described in this paper involving these independently developed systems formed part of the initial work that is now being extended to exploit the significant potential of both systems in more involved cognitive radio experiments.

\section{CTVR Plastic Project Architecture}

The reconfigurable core being used in this joint project is based on a multi-threaded General-Purpose-Processor platform [11], [14]. This system is known as the Plastic Project and has two main objectives in the context of this project. The first main objective of the reconfigurable core is to implement any and all of the required changes in the entire communication stack from the application to physical layer (PHY). The second main objective of the core is to provide awareness information to the cognitive engine [15]. This information may include both internal radio communication system awareness and external radio environment awareness. The cognitive engine may then use this information as a key element of its cognition cycle.

A communications stack is implemented in this highly reconfigurable core as a structure containing a hierarchy of individual processing modules called Components. Considered individually, each Component can implement some or all of the functionality of a signal processing stage of the transceiver signal chain in the PHY and the majority of the other layers in the communications stack. The granularity of a Component is dependent on the designer's needs. In addition to Components existing exclusively in software, the set of Components includes hardware modules with software and firmware interfaces.

Each Component may have a number of parameters associated with it. Examples include a routing layer, which may have a cache size and beacon interval, the RF front-end operating frequencies and power levels, physical layer modulation and coding schemes, and almost any other related aspect of a communications stack that affects its operation. These parameters may be exposed by Components and reconfigured dynamically in order to alter the manner in which those Components operate.

Reconfiguration Control Mechanism: The Plastic Project reconfigurable core communications stack uses a stack manager interface to handle the reconfiguration events and cross-layer dependencies. Together with parametric reconfiguration, the Plastic Project facilitates two additional levels of reconfiguration that serve to fulfill the key objective of implementing change in the communications stack. The second level of reconfiguration is referred to as structural reconfiguration and involves the removal, addition, replacement, or interchange, of individual Components within the Plastic Project structure. Application reconfiguration forms the third and highest level of reconfiguration provided by the Plastic Project and describes the ability to replace, remove, and add entire communications stacks.

In order to fulfill the second main objective of providing awareness information to the cognitive engine, the Plastic Project maintains a shared system database referred to as a blackboard. Each Component within the Plastic Project structure is provided with access to the blackboard in order to expose awareness information that may become available. Examples of such awareness information include the current bit error rate (BER) observed within a decoding Component, the power spectral density (PSD) for the radio channel obtained through the fast Fourier transform (FFT) stage of an orthogonal frequency division multiplexing (OFDM) demodulation component or simply the current modulation scheme complexity being used by the modulation Component of a PHY transmitter signal-chain.

\section{CWT Cognitive Radio Architecture}

The cognitive engine described in this section has been developed at the Center for Wireless Telecommunications 


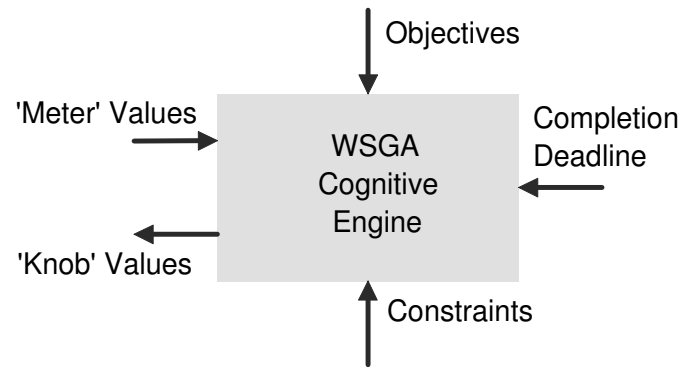

Figure 1. Conceptual diagram of WSGA engine.

(CWT) based in Virginia Tech [4], [5]. This engine implements the awareness-processing, decision-making, and learning elements of cognitive functionality. Specifically, this engine is capable of learning the behaviour of the radio in the different environments over time and intelligently changing the communications stack to new wireless communications scenarios and problems efficiently based on a set of objectives and constraints. A genetic algorithm (GA) approach is used to optimise the communications stack layer parameters. Called the wireless system genetic algorithm (WSGA), this is a powerful method for exploiting the features of the highlyreconfigurable core and optimising the operation of this core across layers in the stack. Fig. 1 illustrates the concept of the WSGA, where the optimisation process produces an output (the value a CR knob should adopt) that attempts to help achieve the desired objectives, based on the input parameters (meter) values and current context, according to a set of constraints (this also includes the time period in which it must be achieved by).

It is important to point out that one of the main objectives of a CR system is to devise and implement a sufficient solution within a specific time period and not an optimal solution after an implementation deadline has expired. For example, consider the example where a cognitive engine is required to deduce a suitable transmitter power level to use within a certain completion deadline ( $7 \mathrm{~ms}$ for example). The cognitive engine may decide that the optimum TX power level is $30.003 \mathrm{dBm}$ but $10 \mathrm{~ms}$ is the time required to produce this result. A sufficient solution may be $-30 \mathrm{dBm}$ or even $-29 \mathrm{dBm}$, and to produce this result, it may only require $5 \mathrm{~ms}$. However, the value of this result is greatly increased because it is a sufficient solution achieved within the specified deadline.

In their original and most basic form, genetic algorithms (GA) were designed as single-objective search and optimisation algorithms. Common to all GAs is the chromosome definition. This dictates how the data are represented, the selection mechanism for choosing the chromosomes that will survive from generation to generation, and the evaluation function used to determine the fitness of a chromosome [5]. The establishment and maintenance of effective wireless communications over a volatile communications channel requires a careful balance of the correct PHY parameters and order in which the signal chain is implemented. This bal- ancing act can be effectively viewed as a complex multidimensional optimisation problem, where the choice of the radio parameters on all layers affects the radio's behaviour in many dimensions including (and not limited to) the bit error rate (BER), transceiver bandwidth, energy consumption, and network latency. Each of these dimensions has some relationship to the set of user and system objectives in mind. In fact, these relationships can also change in their relative importance according to the desired wireless communications application. For example, maintaining low latency in a wireless network is important for multi-player games and audio/video communications; however, for file and short message transfers, the emphasis is generally on maintaining a specified data rate and reliable transportation.

The WSGA is a multi-objective genetic algorithm (MOGA) that can influence the behaviour of a reconfigurable communications stack by modelling the stack as a biological organism and optimising its performance through genetic and evolutionary processes. In the WSGA, radio behaviour is interpreted as a set of layer operation parameters defined by traits encapsulated in the genes of a chromosome. Other general radio functional parameters (including, but not limited to, payload size, power, coding techniques, encryption, equalisation, number of sub-carriers, network protocol, retransmission requests, and spreading technique/code) are also identified as possible genes in the chromosome definition to cater to all of the layers in the communication stack. The WSGA analyses the chromosome's fitness by considering a set of fitness functions defined by performance evaluations of the current communication stack. Each fitness function is weighted to represent the relative importance the user has associated with each objective. The stopping condition for deciding when an optimal or sufficient solution has been obtained is based on the user's quality of service (QoS) and application requirements. Efficiency and optimisation can be subjective quantities; therefore, it is important that to know that over-maximising is essentially a waste of radio resources such as spectrum and energy and a waste of the extra time required to complete the optimisation task(s).

\section{Test Scenarios and Performance Analyses}

This section describes the coexistence scenarios used for the initial tests involving the CWT and CTVR cognitive radio systems.

\section{A. Coexistence Experiments}

The minimal RF front-end used for the over-the-air tests in the coexistence experiments, and for both CR architectures was a $2.3 \mathrm{GHz}-2.9 \mathrm{GHz}$ universal software radio peripheral (USRP) [9], which has a USB 2.0 interface and a $20 \mathrm{~mW}$ maximum transmitter power output to the antenna. This USRP RF front-end performs interpolation/decimation, up/downconversion, signal amplification, and conversion to/from the analog and digital signal domains.

For the coexistence tests, the CWT cognitive radio architecture was configured to act as a narrowband DBPSK modem 


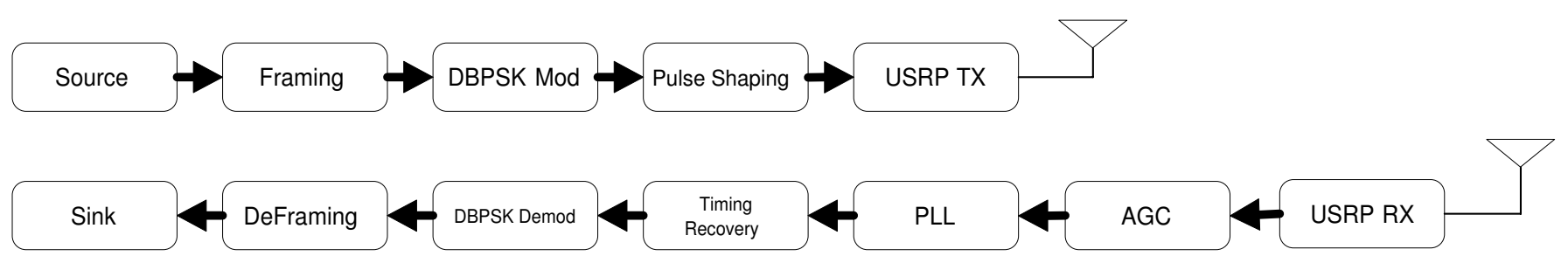

Figure 2. Basic block diagram of the TXRX signal chain used for the CWT and CTVR coexistence tests.

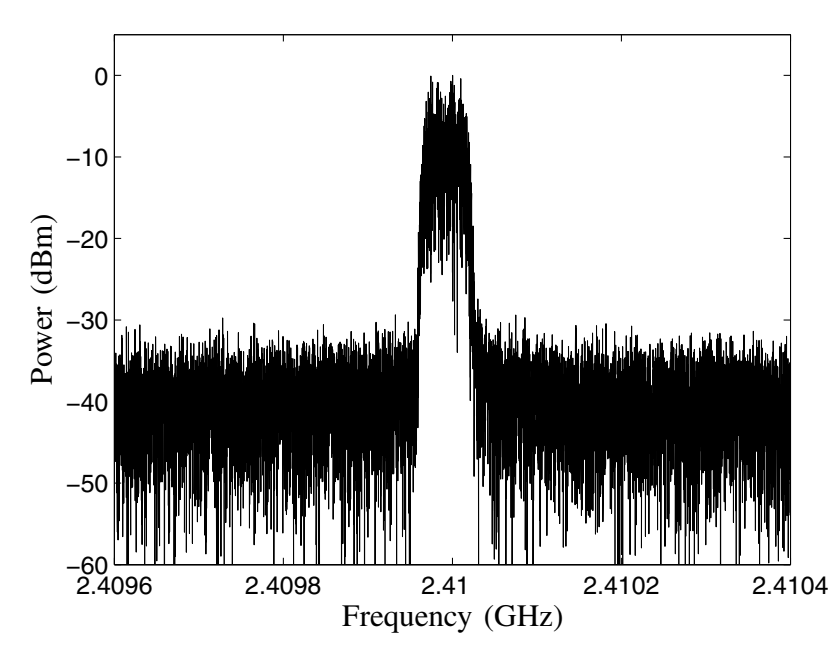

Figure 3. Power spectral density of received primary user DBPSK signal.

operating at a centre frequency of $2.41 \mathrm{GHz}$ and at a data rate of $50 \mathrm{~kb} / \mathrm{s}$. This node was designated as being a primary/legacy user. Fig. 2 illustrates the basic block diagram of the signal chain used by CWT acting as a primary user and reconfigured to create the secondary user transmissions by CTVR. The main objective of this experiment was to attempt to utilise the unused spectrum adjacent to this primary user while minimising the possibility of interference with the primary user. Fig. 3 shows the PSD of the primary user DBPSK signal with no other users/services in the same frequency range observed on the campus of Virginia Tech, Blacksburg, VA, USA during September 2006. The Plastic Project CR system adopted the role as a secondary user attempting to utilise the spectrum whitespace in an opportunistic manner. The secondary user used reconfigurable orthogonal frequency division multiplexing (OFDM) [13] in order to avail of the spectrum white spaces. This involves the following tasks:

- Detection of unused frequency spectrum and avoidance of active primary user frequencies (spectrum whitespace detection).

- Plastic Project reconfiguration to avail of the unused spectrum (exploiting available spectrum).

- Synchronisation with other secondary user nodes.

1) Spectrum whitespace detection and exploitation: For the initial coexistence experiments, fixed subcarrier indices were used in order to measure the immediate effects of the secondary user interference on the primary user. This developing project is taking the following approach regarding the implementation of this scheme in a more dynamic context: The detection of unused spectrum within the accessible bandwidth of the transceiver is accomplished using an energy detection and threshold scheme. This feature is based on prior work that may be found in [12]. Essentially, each secondary user obtains a periodogram of the band of interest. Periodograms are derived from the FFT stage of Plastic Project secondary user node and enables the power spectral density of the band of interest to be extracted without a significant increase in the demand for signal processing resources.

Specifically, the periodogram information, denoted by $M(k)$ in Eq. 1 is obtained by calculating $L$ estimates of the power spectral density (PSD), $Y$ and averaging:

$$
M(k)=\frac{1}{L} \sum_{n=0}^{L-1}\left|Y_{n}(k)\right|^{2}
$$

A list of available frequencies are compiled from the periodogram bin numbers corresponding to frequencies where the detected power level is less than the threshold value. A reduced complexity channel mask is constructed enabling blocks of available frequencies to be grouped and represented as binary values. Further information regarding this scheme may be found in [12]. This developing channel mask is being further refined minimise the overhead that must be incurred when conveying the spectrum usage information to other remote secondary user nodes. Using this information, the Plastic Project CR device can reconfigure to take advantage of this estimate of the unused frequencies, on either side of the primary user DBPSK signal by changing the subcarriers employed in the orthogonal frequency division multiplexing stage in the transmit signal chain. Fig. 4 shows the reconfigurable OFDM signal occupying unused spectrum on either side of the primary user DBPSK signal. In this case, guard bands were used to separate the primary and secondary users. The DBPSK signal in this case was received with zero packet errors in a test involving the transmission of 2000 packets. Fig. 5 shows the primary user using DBPSK (CWT) and secondary user using OFDM (CTVR) with no guard bands separating the two different services. In this case, $100 \%$ packet loss was experienced by the primary user service and the power of the secondary user had to be reduced to ensure at least $20 \mathrm{~dB}$ SNIR before the primary user experienced reduced packet loss.

The ability to coexist in an interference-free manner is 


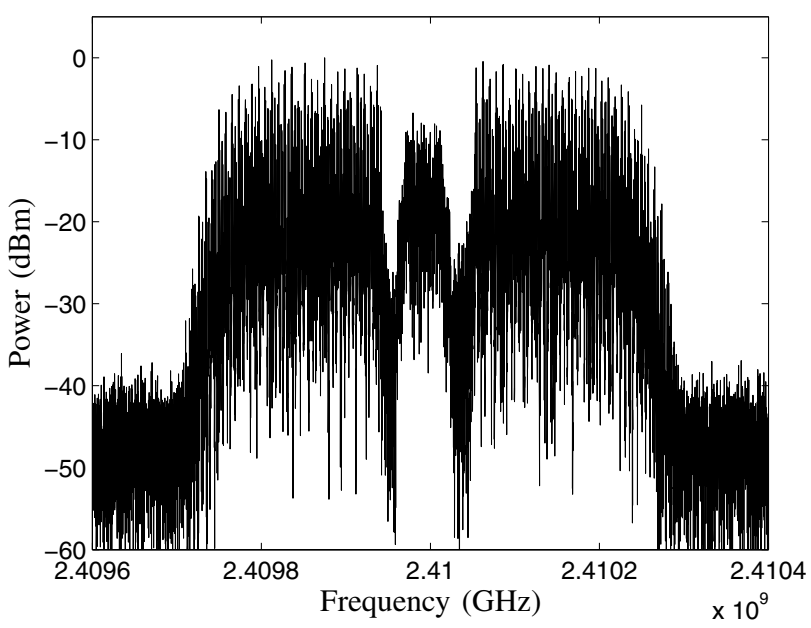

Figure 4. Power spectral density of received primary (DBPSK) and secondary user (OFDM) signals coexisting in the same frequency band with guard bands between the primary and secondary users.

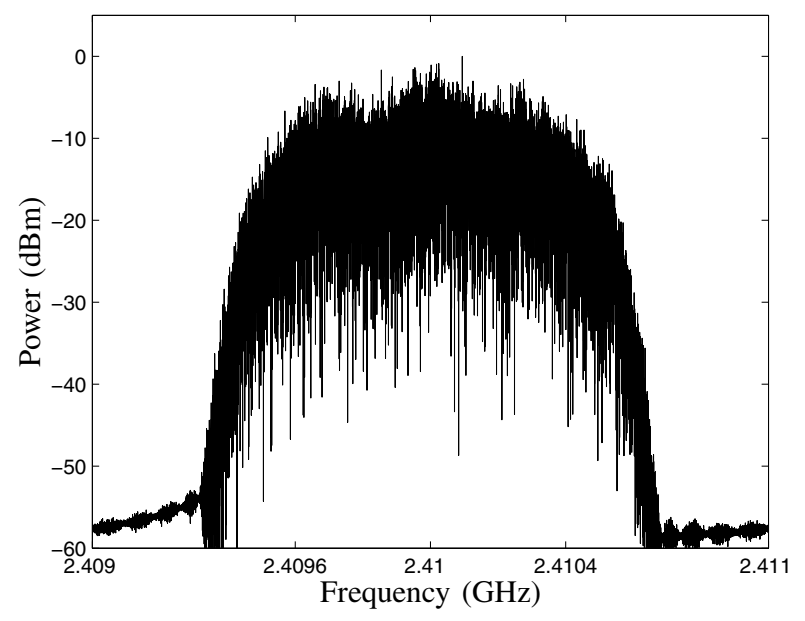

Figure 5. Power spectral density of received primary (DBPSK) and secondary user (OFDM) signals coexisting in the same frequency band with no guard bands separating the two users. The secondary user is interfering with the primary user in this case.

heavily dependent on the ability of an opportunistic user to accurately detect presence and bandwidth of an active primary/legacy user. Fig. 6 illustrates the PSD of the same DPSK primary user signal as for the first test case, but where the OFDM signal originating from the secondary user activity is now interfering with the primary user. In this scenario involving a 2000 packet transmission test by the primary user, 185 packets were received incorrectly. This represents a $9.25 \%$ packet loss resulting from secondary user interference. This example helps to illustrate the importance of a high reaction speed for secondary opportunistic users who can have a detrimental effect on the primary user if unable to immediately vacate newly occupied spectrum.

The main purpose of these tests was to show that the

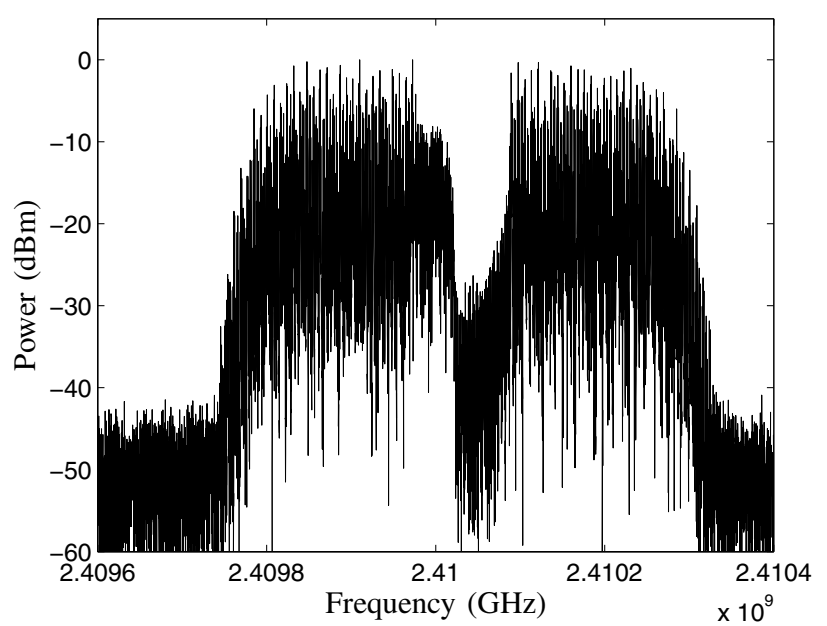

Figure 6. Power spectral density of received primary (DBPSK) and secondary user (OFDM) signals, where the secondary user signal is interfering with the primary user.

concepts of interoperability and interference-free coexistence are feasible. However, a node wishing to exploit these features should be able to accurately detect and characterise spectrum whitespace, take advantage of these opportunities, and then coordinate with the destination secondary user nodes. These three key challenges require robust solutions in order to encourage the adoption of this technology in licensed primary user spectrum regimes.

\section{CONCLUSIONS}

This paper has described some of the initial key results from a collaborative cognitive radio and networks project involving the Centre for Telecommunications Value-Chain Research (CTVR) in Ireland, and the Center for Wireless Telecommunications (CWT) in the U.S. These results concern the abilities of different software-defined radio (SDR) and cognitive radio (CR) systems to coexist in common frequency bands. Initial analysis of coexistence experiences involving primary users and secondary opportunistic spectrum users in a common frequency band have also been presented. In this case, interference-free and interfering cases were analysed and it was shown that for the worst case scenario where there were no guard bands between the primary and secondary users, the primary user was minimally affected by the opportunistic secondary user transmissions.

\section{ACKNOWLEDGEMENTS}

This material is based upon work supported by Science Foundation Ireland under Grant No. 03/CE3/I405 as part of the Centre for Telecommunications Value-Chain Research (CTVR) at Trinity College Dublin, Ireland. This work was also supported by the National Science Foundation under grants 9983463, DGE-9987586, and CNS-0519959 and by the National Institute of Justice, Office of Justice Programs, US Department of Justice under Award No. 2005-IJ-CX-K017. 
The opinions, findings, and conclusions or recommendations expressed are those of the author(s) and do not necessarily reflect the views of the National Science Foundation or the Department of Justice.

\section{REFERENCES}

[1] Nolan, K. E., Rondeau T.W., Sutton, P.D., Bostian, C. W., Doyle, L.E., "A Framework For Implementing Cognitive Functionality", in Proceedings of the 51st SDR Forum General Meeting and 2006 Technical Conference, Nov. 13-17, 2006, Orlando, Florida.

[2] Nolan, K. E., Rondeau T.W., Sutton, P.D., Le, B., Bostian, C. W., Doyle, L.E., "Demonstration and Analyses of Collaboration, Coexistence, and Interoperability of Cognitive Radio Platforms" (Invited paper), in Proceedings of the 1st IEEE Workshop on Cognitive Radio Networks, Jan 11th, 2007, Las Vegas, NV.

[3] Haykin S., "Cognitive Radio: Brain-Empowered Wireless Communications", IEEE Journal on Selected Areas in Communications, February, 2005.

[4] T. W. Rondeau, B. Le, D. Maldonado, D. Scaperoth, A. B. MacKenzie, and C. W. Bostian, "Optimization, Learning, and Decision Making in a Cognitive Engine," in Proc. SDR Forum 06 Technical Conference, Orlando, FL, 2006.

[5] Fette, B. (Editor), Cognitive Radio Technology, Newnes, Aug. 2006,ISBN: 0-7506-7952-2

[6] Doyle, L.E., Nolan, K.E., Forde T.K. et al., "A Platform for Dynamic Spectrum Experimentation", in Proceedings of the 1st International Workshop on Technology and Policy for Accessing Spectrum (TAPAS), Boston, August 1-5 2006
[7] T. W. Rondeau, C. J. Rieser, B. Le, and C. W. Bostian, "Cognitive Radios with Genetic Algorithms: Intelligent Control of Software Defined Radios," SDR Forum Technical Conference, Phoenix , 2004, pp. C-3 C-8.

[8] Rieser, C.J.; Rondeau, T.W.; Bostian, C.W.; Gallagher, T.M., "Cognitive radio testbed: further details and testing of a distributed genetic algorithm based cognitive engine for programmable radios," Military Communications Conference, 2004. MILCOM 2004. IEEE, vol.3, pp. 1437- 1443, 31 Oct.-3 Nov. 2004.

[9] Universal Software Radio Peripheral (USRP) by Ettus Research LLC. Further information is available at http://www.ettus.com/

[10] The GNU Software Radio Project. Further information is available at http://gnuradio.org/trac

[11] P. Mackenzie, Reconfigurable Software Radio Systems, Ph.D. dissertation, University of Dublin, Trinity College, 2004.

[12] Nolan, K.E., Doyle, L.,Mackenzie, P., O Mahony, D., "Fluid Wireless -Dynamic Spectrum Allocation and Spectrum-Monitoring Application Using Reconfigurable Radio and OFDM", in Proceedings of the 2005 Software Defined Radio Forum (SDR Forum) Technical Conference, Anaheim, CA, USA, November 14-18, 2005.

[13] Nolan, K.E., Reconfigurable OFDM Systems, Ph.D. dissertation, University of Dublin, Trinity College, 2005.

[14] Sutton, P., Doyle, L., Nolan, K.E.,"A Reconfigurable Platform for Cognitive Networks", in Proceedings of the 1st International Conference on Cognitive Radio Oriented Wireless Networks and Communications (CROWNCOM 2006), June 8-10, 2006.

[15] Nolan, K.E., Sutton, P., Doyle, L., "An Encapsulation for Reasoning, Learning, Knowledge Representation, and Reconfiguration Cognitive Radio Elements", in Proceedings of the 1st International Conference on Cognitive Radio Oriented Wireless Networks and Communications (CROWNCOM 2006), Greece, June 8-10, 2006 